\title{
A Research on Synergic and Spiral-Propulsion Mechanism of Enterprise Information Systems Growth
}

\author{
Xinhua Bi and Cuiling Yu \\ School of Management, Jilin University, Changchun 130022, P.R. China \\ fasthome@yeah.net yucuiling0908@sina.com
}

\begin{abstract}
The mechanism of information system (IS) growth is an important part of IS growth theory and has attracted much attention of scholars and entrepreneurs. As validated by many studies, the process of IS growth is influenced by various factors within the enterprise and from external environment. The external factors are mainly governmental propulsion, competitive pressure, linkage demand of cooperative enterprises and IT suppliers' propulsion. The internal factors are top management support, middle management support, IT department propulsion and other organizational personnel's cooperation and participation. The synergic effect of internal and external factors promotes the development of IS construction and the growth of enterprise information systems. This paper pays more attention to the microcosmic mechanism of IS growth and proposes that IT absorption is a direct impetus to IS growth. It analyzes synergic effect of the subjects and elements of IT absorption. Furthermore, a mathematic model is used to describe the spiral-propulsion mode of IS growth. Viewed from a holistic perspective, IS growth presents itself as a dynamic and spiral process.
\end{abstract}

Keywords: Enterprise information systems (EIS), Information systems growth (IS growth), Synergic and spiral-propulsion mechanism, Information technology absorption (IT absorption)

\section{INTRODUCTION}

With increasing drastic commercial competition, multiplex customer demand and expedite change speed, it has become a hot topic in information system (IS) research field that how organizations utilize information technology (IT) to improve its competence continuously and achieve competitive advantages [1-2]. Therefore, IS growth theory has attracted much attention of scholars and entrepreneurs. IS growth is characterized as a continuously rising process in which IS application evolves from lower stage to higher, from simplicity to complexity, from local implication to systematic integration [3]. Many studies have demonstrated that IS growth is influenced by various factors within the organization and from external environment. The complexity of growth process requires mutual coordination and cooperation of different power to reach maximum synergy and promote enterprise information systems to evolve to higher stages. The whole process presents itself as a spiral mode.

Please use the following format when citing this chapter:

Bi, X., Yu, C., 2007, in IFIP International Federation for Information Processing. Volume 254, Research and Practical Issues of Enterprise Information Systems II Volume 1, eds. I.. Xu, Tjoa A., Chaudhry S. (Boston: Springer), pp. $797-$ 806. 
Viewed from microcosmic level, enterprise information systems stem from a series of IS construction engineering which enable it to gain continual growth through absorbing new technologies. Within the enterprise, nonlinear interactions of the subjects and various elements related to information systems engender state fluctuation which produces order parameters promoting IS construction. In fact, the introduction of an IS project is a great fluctuation caused by magnification of the nonlinear interactions. Using the method of theory analysis together with mathematic model, this paper tries to make a further analysis on the synergic and spiralpropulsion mechanism of IS growth.

\section{THE SYNERGIC AND SPIRAL-PROPULSION MECHANISM OF IS GROWTH}

\subsection{A Synergic and Spiral-Propulsion Mechanism Model of IS Growth}

Many studies have demonstrated that IS growth is influenced by factors both from internal and external environment of an enterprise. Since there are interactions of lots of subsystems during the synergy process, the synergic equation would be very complex because of the impact of too many variables. However, servo principle provides us with a practical and operable methodology which suggests that it needn't to concern all variables and factors but to grasp the most primary and influential variables and neglect minor and trivial variables, thus to approach order state step by step [4]. Based on this idea, we divide these key factors into two classes according to the subjects of this process. The first is external factors, including governmental propulsion, competitive pressure, linkage demand of cooperative enterprises and IT suppliers' support. The second is internal factors, including top management support, middle management support, IT department propulsion and other organizational personnel's participation and cooperation.

The action mechanism of these factors on IS construction is shown as Figure 1. External power refers to the impact of external factors on IS construction. Internal power refers to the impact of internal factors. According to synergic principle, with the increasing strengthening of external parameters, viz. propulsion of governmental policy, informationization act of competitors, linkage demand of supply chain and propulsion of IT supplier, enterprise state of production and operation would be effected, which would changes the main conflicts of the enterprise, causing the organizational system to become unstable. Enterprise must seek a new ordered structure to adapt external environment. While IT solution helps to satisfy this change demand, organizational leader would be motivated to adopt the technology. The synergic effect of top management, middle management, IT department and other organizational personnel produces internal propulsion for information systems. Furthermore, under the guidance of attractors, it evolves into a cohesion which makes various power synergize in the direction of IS construction and promote IS growth. 


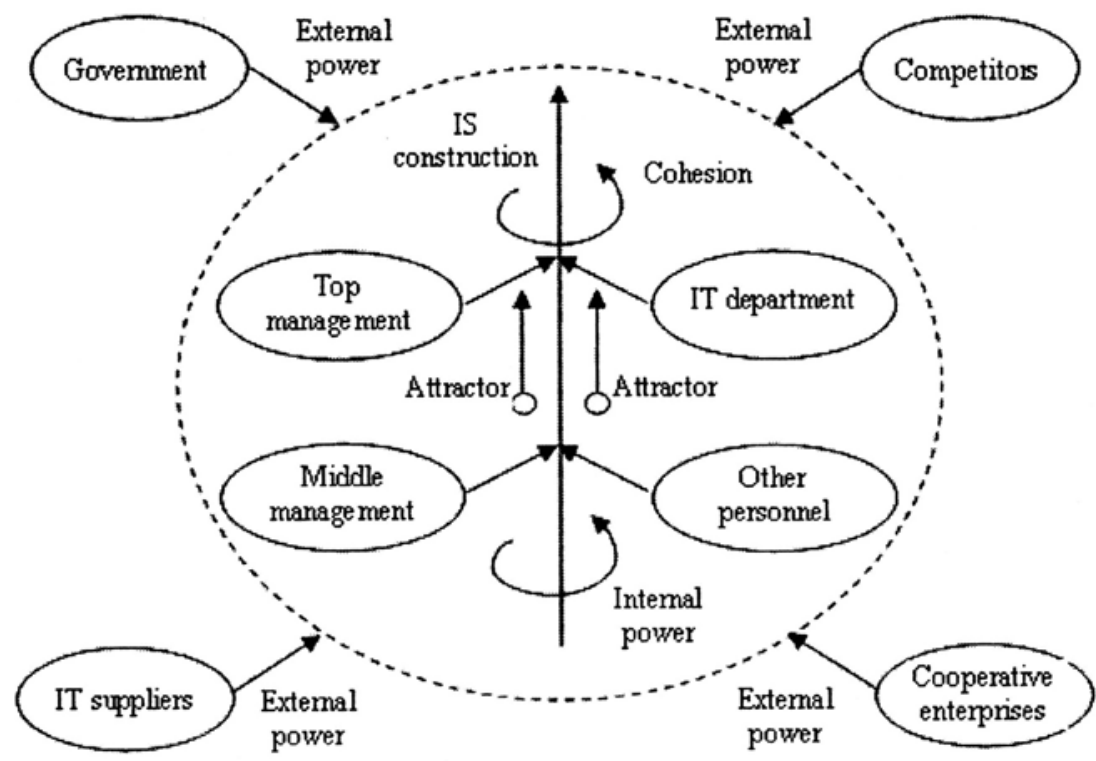

Figure 1. A Synergic and Spiral-Propulsion Mechanism Model of IS Growth

This paper emphasizes on the mechanism of IS growth at microcosmic level. For an enterprise, IS growth stems from a series of IS construction engineering, which conforms to a fundamental law that it is a process beginning from system analysis to system design, then system implementation, till system application. Viewed from a dynamic behavioral perspective, it is a process in which the organization identifies, adopts, adapts, accepts and infuses related IT solution. It is called IT absorption which has been addressed as a special topic by the authors [5]. Based on the stage model of IT implementation proposed by Kwon and Cooper [6-7], a process model of IT absorption is developed which describes IT absorption as a dynamic closed loop composed of five stages, viz. identification, adoption, adaptation, acceptance and infusion, and it integrates a role of "knowledge base" into these five stages.

The process of IT absorption begins from identifying enterprise demand and corresponding IT solutions. Then the enterprise evaluates and compares these solutions to make an adoption decision of whether to make an investment, and puts the one adopted into practice. The most important thing in the next stage is to achieve well mutual adaptation between the organization and information technology. Gradually, organizational personnel accept it through practice and learning. Then IT application spreads and diffuses continuously until the usage of it becomes a conventional activity in the organization. Consequently, information technology infuses into the organization. These five stages proceed and circulate unceasingly, forming a closed loop. The "knowledge base" located in the middle of the model interacts with the five stages and external environment, enabling organizational comprehension of related IT knowledge and experience to accumulate and increase ceaselessly. Viewed from an initiative process perspective, the essential of IS growth 
is a spiral process propelled by these five stages. Therefore, it can be regarded that the process of IT absorption reflects the microcosmic mechanism of IS growth. Hereby, a microcosmic mechanism model of IS growth is developed as shown in Figure 2.

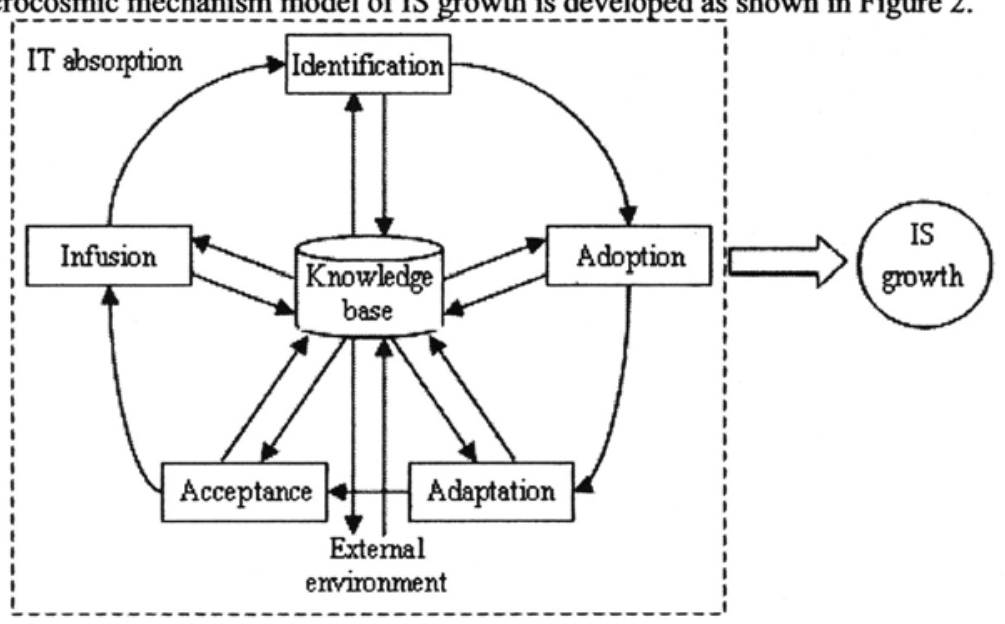

Figure 2. A Microcosmic Mechanism Model of IS Growth

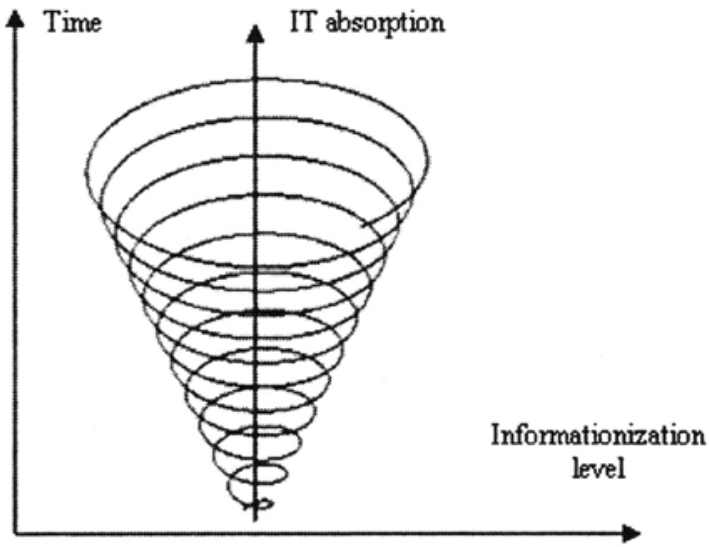

Figure 3. A Spiral Model of IS Growth

The synergic effect of various powers within the enterprise impels it to absorb and utilize information technology effectively. With the development of business strategy, enterprise may introduce new information technology and begin another circle of IT absorption. This process circulates continuously, and every circulation is based on experience accumulation of the prior. Viewed from a holistic perspective, IS growth presents itself as a dynamic and spiral process as shown in Figure 3. 


\subsection{Subjects Synergy in IS Growth Process}

IT absorption is a complicated system engineering related to top management, middle management, IT department and other organizational personnel of the enterprise. We call these key personnel as the subjects of IT absorption. Cooperation and synchronization requirements of IS application demand that all subjects examine their functions from a perspective of maximizing the holistic benefit.

In the stage of identification, management of the enterprise should observantly apperceive enterprise problems, environmental changes and the trends of IT development to set down corresponding IT solutions. Simultaneity, it needs the cooperation of IT department and all organizational personnel.

In the stage of adoption, the decision-maker must consider demands put forward by personnel of all departments; otherwise the adopted solution probably might be difficult to implement and spread. Moreover, management of the enterprise should provide necessary resources IT department

In the stage of adaptation, IT department cooperates with software provider to implement information system and provides training to organizational employee. At the same time, enterprise management needs to coordinate and control the conflicts and contradiction to make the organization synergize with IT solution.

In the stage of acceptance, management of the enterprise should make a good communication with employee to motivate them to convert inherent idea and behavioral inertia to support IT application. IT department should provide technological training for organizational personnel and help them master related knowledge and skills.

In the stage of infusion, IT department needs to make continuous improvement for information systems to realize more comprehensive and integrated application. Management of the enterprise needs to cultivate "IT culture" to infuse information technology into the organization.

Knowledge management needs collaboration and cooperation of all organizational personnel which can promote knowledge accumulation, share, conversion and protection, thus to gain continuous accumulation of IT absorptive capacity.

\subsection{Elements Synergy in IS Growth Process}

IS growth is a complicated nonlinear process, also a system with various elements which need comprehensive management. G. Zheng proposed a diamond model for comprehensive synergy of innovation elements and pointed out six critical elements of innovation process, viz. technology, strategy, market, organization, culture and institution [8]. Using his viewpoints for reference, this paper proposes six crucial elements at microcosmic level, viz. technology, strategy, organization, culture, personnel and institution.

For various elements, their categories, manners and means to acquire information and energy from external environment are different, and they are distinct in the intensity, intention and methods of their effect in different conditions or stages, fluctuation and conflict inevitably exist in the system. To realize the evolution from disorder (beginning from IT introduction) to order (infusing information technology 
into organization), various elements interact with each other and finally engender order parameters which propel information system to evolve into advanced state. Therefore, it needs good synergy of all elements.

At technology aspect, the introduced IT solution must meet enterprise demand. Meanwhile, technology must synergize with other elements to impel IT absorption. Many studies have indicated that the primary failure reason of most IS projects is not technology but lies in its ineffective coordination with non-technology elements including strategy, culture, organization, personnel and institution.

At strategy aspect, information system planning (ISP) should match with business planning (BP). Lack of ISP or Lack of match of ISP with BP usually causes shortterm benefit oriented, sightless and random decision, which make it difficult to achieve expected goal of IS construction. Only under the guidance of ISP could all elements run effectively and ensure the correctness of synergic direction.

It is needed to adjust and optimize organizational structure and business process during the process of IS implementation. Disordered hierarchy, intersected structure and slack business process impact implementation process and the performance of IT application. Therefore, it is needed to establish a structure which can support elements synergy and enhance coordination of different departments and function units.

To IT application, corporate culture is a two-edge sword which impacts the whole process of IT absorption inherently. On one hand, IT application can gain more support and positive participation in an innovative and adventurous culture; On the other hand, IT application would suffer resistance if it threatens current culture.

IS construction is a complicated process that relates to all organizational personnel. Viewed from a dynamic perspective, the main factors which directly determine the impetus are the subjects and their actions in this process, that is, the personnel concerned with information system. So, organizational personnel have a quite active impact on IS construction.

Institution provides concrete guarantee mechanism and tool to adjust and control the behaviors of organizational personnel, and to solidify related experience and fruit of IS construction.

Comprehensive synergy of all these elements guarantees the success of IS construction. The essence of synergy is multi-coordination which enables them to exert advantage and achieve symbiosis and coexistence, thus to promote IS growth.

\section{A MATHEMATICAL MODEL}

As mentioned in section 2, there are complex relations of the factors during the process of IS growth, which may be promotion or restraint. When information systems become more and more advanced and complex, its improvement would become more and more difficult. For the diversity of factors, complexity of system and increase of difficult in the process of IS growth, it can be regarded as a SelfIncreasing-Difficulty System (SIDIS), and the effective strategy for its development is "Spiral Combining Propulsion Principle" (SPIPRO principle) [9]. Viewed from system science, it is a spiral process driven by synergic effect of critical factors and can be reflected by improvement of informationization level. Using the SPIPRO 
mode which describes technological catching-up and technological leapfrogging for reference [10], this paper makes a further analysis on the synergic and spiralpropulsion mechanism of IS growth with a mathematic model.

\subsection{Conception Definition}

Definition 1. When enterprise introduces an information technology/information system, enterprise information system would gain a growth if IS construction is successful, that is, it absorbs the technology successfully. We use $P(t)$ to represent the probability of achieving a success of IS construction, $P(t) \in[0,1]$. We hypothesize that the probability of achieving a success of IS construction is greater while $P(t)$ is larger.

Definition 2. We propose the conception of absorptive capacity for information technology which expresses organizational abilities of identification, adoption, adaptation, acceptance, infusion and knowledge management for IT solution in the process of IT absorption, and use $A(t)$ to represent it.

Definition 3 . We use $D(t)$ to represent system difficulty which must be conquered when enterprise constructs information systems at time $t$.

Definition 4. We hypothesize that technological level of enterprise informationization at time $t$ is $a(t)$ and that of expected after adopting new technology is $b(t) ; a(t)$ and $b(t)$ are monotonic increasing function. Therefore, the result of subtracting $a(t)$ from $b(t)$ is technological gap marked by $h(t), h(t)=b(t)-a(t)$. In fact, IS construction changes the logic and efficiency of information management and is also a reengineering for enterprise system, which is represented by improvement of economic effectiveness and organizational order of the enterprise [11]. $m(t)$ is used to represent economic effectiveness and $n(t)$ is used to represent organizational order at time $t$. We take $r(t)$ to record the total gap of informationization level between that of original and expected, $r(t)$ is the sum of $h(t), m(t)$ and $n(t)$.

\subsection{Mathematic Model and Its Meanings}

The following mathematic model is used to describe the synergic and spiralpropulsion path of IS growth.

$$
\begin{gathered}
\rho=\frac{e P(t)}{1-e \cos (w(t) t)} \\
S(t)=\frac{1}{2} \int \rho^{2} \omega(x) d x
\end{gathered}
$$

Equation (1) refers to accumulative plane equation and equation (2) refers to accumulative area equation. The two are used to describe the "spiral" path of IS growth. Their meanings are: the projection of the path of IS growth on $x-y$ plane is a cone curve; $x-y$ is accumulative plane and the enclosed cone curve forms accumulative area. 
In equation (1), e refers to the eccentricity. The actual input of IS construction is absolutely consistent with its goal if $\mathrm{e}=0$. But there is a departure from expected direction if $e \geq 1$. $\omega$ refers to the accumulative speed in the spiral process. It is represented by the functions of critical factors of IS construction, $w(t)=f($ technology, strategy, organization, culture, personnel, institution). On the other hand, viewed from the subjects of IT absorption, $w(t)=f($ top management, middle management, IT department, other organizational personnel).

It is hypothesized that system difficulty at time $t$ positively correlates with the gap of informationization level which is embodied by technology level, economic effectiveness and organizational order. It is represented by equation (3).

$$
D(t)=\left\{\begin{array}{ll}
r(t) & r(t)>0 \\
0 & r(t) \leq 0
\end{array}, r(t)=h(t)+m(t)+n(t)\right.
$$

\subsection{Description of the Synergic and Spiral-Propulsion Path}

For the "spiral" process, it is important to accumulate absorptive capacity of information technology. The area enclosed by curve projection on accumulative plane is marked by $\mathrm{S} . S=S(t)=\frac{1}{2} \int \rho^{2} \omega d t, \mathrm{~S}(0)=0$. We hypothesize that $\mathrm{S}\left(\mathrm{t}_{0}\right)=\mathrm{S}_{0}, \mathrm{~S}_{0}$ is a threshold which is the area necessary to accumulate for the enterprise. In this process, it is necessary to complete absorptive capacity accumulation of all aspects, including identification, adoption, adaptation, acceptance, infusion and knowledge management. The projection curve moves on the plane $\mathrm{Z}=\mathrm{Z}_{0}$ during the spiral process. Only when the accumulation of absorptive capacity reaches certain extent could enterprise information systems gain growth. Enterprise completes accumulation process when $S=S_{0}$. Therefore, only when the accumulation of absorptive capacity exceeds the threshold $S_{0}$, that is, when $\frac{1}{2} \int_{0} \rho^{2} \omega(x) d x>S_{0}$, could enterprise information system gain "propulsion" following "spiral"; when $\frac{1}{2} \int_{0} \rho^{2} \omega(x) d x \leq S_{0}$, there is only "spiral" but no "propulsion". It is necessary to accelerate the speed of capacity accumulation in order to shorten the time for accumulation. However, only a good synergy among various elements of this process can enable absorptive capacity to accumulate continuously and promote IS construction.

During the process of information system construction, the probability of success correlates positively with IT absorptive capacity of the enterprise while correlates negatively with system difficulty which depends on not only the progress of time but also the gap of informationization level. Therefore, it is hypothesized that

$$
P(t)=\frac{A(t)}{D(r(t), t)}
$$

Differential coefficient of $P(t)$ is that 


$$
\begin{aligned}
\frac{d P(t)}{d t} & =\frac{\frac{d A(t)}{d t} D(r(t), t)-\frac{d r(t)}{d t} \frac{\partial D(r(t), t)}{\partial r(t)} A(t)-\frac{\partial D(r(t), t)}{\partial t} A(t)}{D(r(t), t)^{2}} \\
& =\frac{1}{D(r(t), t)} \frac{d A(t)}{d t}\left(1-\varepsilon_{r}-\varepsilon_{t}\right)
\end{aligned}
$$

In equation (5),

$\varepsilon_{r}=\frac{\partial D(r(t), t)}{\partial r(t)} \frac{A(t)}{D(r(t), t)} \frac{d r(t)}{d A(t)}$, and $\varepsilon_{\mathrm{T}}$ refers to the elasticity of system difficulty to informationization level gap;

$$
\varepsilon_{t}=\frac{\frac{\partial D(r(t), t)}{\partial t}}{\frac{d A(t)}{d t}} \frac{A(t)}{D(r(t), t)}, \text { and } \varepsilon_{t} \text { refers to the relative change of system difficulty }
$$

to IT absorptive capacity through time.

Equation (5) indicates that, when $\frac{d A(t)}{d t}\left(1-\varepsilon_{r}-\varepsilon_{t}\right)>0$, the differential coefficient of $P(t)$ exceeds 0 , which means an increase of the probability for IS growth. It is deduced that $\mathrm{P}(\mathrm{t})$ is larger when $\varepsilon_{\mathrm{T}}$ and $\varepsilon_{\mathrm{h}}$ are smaller. Therefore, for the elasticity of system difficulty to informationization level gap and the relative change of system difficulty through time, the smaller, the better; but for the relative change of IT absorptive capacity through time, the larger, the better. Since informationization level gap is objective and system difficulty engendered by it is uncontrollable, only IT absorptive capacity is controllable. Therefore, enterprise should attach great importance to strengthen its absorptive capacity, thus to increase the probability of success for IS construction and promote IS growth.

Many studies have demonstrated that information system growth presents as an " $S$ " curve, which just validates that it is a spiral process. This paper uses a mathematical model to describe the synergic and spiral-propulsion path of IS growth. Through it we conclude that only when it possesses IT absorptive capacity at some extent could enterprise assimilates advanced technology effectively thus to propel IS growth. The data from our investigation also validate the synergic and spiral-propulsion model of IS growth. Using this model and considering the especial condition of its own, enterprise can find out the critical factors of IS construction and impel their synergy and cooperation, thus to improve the accumulative speed of absorptive capacity and promote the growth of information systems.

\section{CONCLUSIONS}

This paper summarizes the factors which influence the growing process of enterprise information systems and develops a model for synergic and spiralpropulsion mechanism of IS growth. It proposes that the synergic effect of internal 
and external factors enables IS construction to advance continuously thus to promote IS growth. Viewed from a holistic perspective, IS growth presents itself as a dynamical and spiral process. This paper emphasizes research on the microcosmic mechanism of IS growth and proposes that IT absorption is the direct impetus to it. A detailed analysis is made on subjects synergy and elements synergy of the enterprise in the process of IS construction. A mathematic model is used to describe the synergic and spiral-propulsion mode of IS growth. It concluded that enterprise should attach great importance to strengthen its absorptive capacity thus to increase the probability of success for IS construction and promote IS growth. This research work is likely to shed light on the mechanism of enterprise information system growth at some extent and provide theoretical foundations and instructions for IS construction.

\section{ACKNOWLEDGEMENTS}

This research was supported by the National Natural Science Foundation of China under Grant 70471014.

\section{REFERENCES}

1. F.J. Mata, W.L. Fuerst, and J.B. Barney, Information technology and sustained competitive advantage: A resource-based analysis, MIS Quarterly. Volume 19, Number 4, pp. 487-505, (1995).

2. E.K. Clemons, R.M. Dewan, and R.J. Kauffman, Special issues: competitive strategy, economics, and information systems, Journal of Management Information Systems. Volume 21, Number 2, pp.5, (2004).

3. X. Bi, W. Shang, and Y. Xu, Comprehensive and comparative analysis on the models of IS growth theory, Information Science. Volume 23, Number 11, pp.1601-1605, (2005).

4. H. Haken, Advanced synergetics (Springer-Verlag: Berlin, 1983).

5. $\mathrm{X}$. Bi and $\mathrm{C}$. Yu, Research on absorptive capacity of information technology and its process model, Science of Science and Management of S.\&T.. Number 12, pp.42-46, (2006).

6. T.H. Kwon and R.W. Zumd, Unifying the fragmented models of information systems implementation, in Critical Issues in Information Systems Research, eds. R. J. Boland and R.A. Hirscheim (John Wiley: New York, NY, 1987), pp.227-252.

7. R.B. Cooper and R.W. Zumd, Information technology implementation research: A technological diffusion approach, Management Science. Volume 36, Number 2, pp.123139, (1990).

8. G. Zheng, TIM based research on the total synergy mechanism of all the innovation agents during innovation process. Ph.D Thesis, Zhejiang University (2004).

9. H. Wang, A kind of systems methodology-the SPIPRO principle, Systems Engineering. Volume 12. Number 5, pp.9-12, (1994).

10. D. Chen, SPIPRO mode research from catching-up to leapfrogging, Studies in Science of Science. Volume 24, Number S, pp.67-73, (2006).

11. $\mathrm{X}$. Bi, Enterprise information systems construction and management change (Jilin University Press: Changchun, Jilin, 2002). 